\title{
Multidimensionally Resolved Pore Size Distributions
}

\author{
J.H. Strange, J.B.W. Webber
}

October 21, 1996

Physics Laboratory, University of Kent, Canterbury CT2 7NR, United Kingdom.

(for AMR)

$\sim 4000$ words

\begin{abstract}
A novel method of determining median pore size and pore size distributions as a function of spatial position inside a porous sample is described.

Pore sizes have been measured with 1, 2 and 3-dimensional spatial resolution, using NMR cryoporometry in conjunction with magnetic resonance imaging techniques.

The method is suitable for pore diameters in the range of $30 \AA$ to over $2000 \AA$ pore diameter, and is based on the technique of freezing a liquid in the pores and measuring the melting temperature by Nuclear Magnetic Resonance. Since the melting point is depressed for crystals of small size, the melting point depression gives a measurement of pore size.
\end{abstract}




\section{NMR Cryoporometry}

Porous materials occur widely and have many important applications. They can, for example, offer a convenient method of imposing fine structure on adsorbed materials. They can be used as substrates to support catalysts and can act as highly selective sieves or cages that only allow access to molecules up to a certain size. Many biologically active materials are porous.

Commercially important porous materials include the porous glasses, the alumina and alumino silicates such as clays and zeolites, activated and other porous carbons, and water and oil bearing shales and limestones, as well as many construction and engineering materials. Porous materials play an important part in many biological processes and life forms. Characterisation and measurement of porous media is therefore of considerable interest.

We may define porosity as the fraction of the sample volume occupied by pore void; an important characterising parameter is the porosity as a function of pore diameter and spatial location, i.e. the poresize distribution within a material.

Recent work by Jackson and McKenna [1] demonstrated that the melting point depression of a variety of liquids confined in silica glasses varied inversely with the mean pore diameter as determined by gas adsorption isotherm measurements.

This behaviour is closely related to the capillary effect; both reflect the change in free energy caused by the curvature of the liquid surface.

The Gibbs-Thomson equation for the melting point depression $\Delta \mathrm{T}_{m}$ for a small crystal of diameter $\mathrm{D}$ is given ${ }^{[1,2]}$ by :

$$
\begin{aligned}
\Delta \mathbf{T}_{m}= & \mathbf{T}_{m}-\mathbf{T}_{m}(\mathbf{D})=4 \boldsymbol{\sigma}_{s l} \mathbf{T}_{m} /\left(\mathbf{D} \Delta \mathbf{H}_{f} \boldsymbol{\rho}_{s}\right) \\
\text { where : } & =\text { normal melting point of bulk liquid } \\
\mathrm{T}_{m} & =\text { melting point of crystals of diameter D } \\
\mathrm{T}_{m}(\mathrm{D}) & =\text { surface energy at the liquid-solid interface } \\
\sigma_{s l} & =\text { bulk enthalpy of fusion } \\
\Delta \mathrm{H}_{f} & =\text { density of the solid } \\
\rho_{s} & =\text { denter to }
\end{aligned}
$$

To exploit this effect for pore size measurement a porous sample containing a liquid is cooled until all the liquid is frozen, and then gradually warmed while monitoring the amplitude of the NMR proton spin echo from any liquid present. The liquid is usually chosen to be water or cyclohexane, the latter offering the large melting point depression of $1825 \mathrm{KA}$. i.e. a depression of nearly $20 \mathrm{~K}$ in $100 \AA$ pores.

NMR is a sensitive technique for distinguishing between solid and liquid, as the coherent transverse nuclear spin magnetisation decays much more rapidly in a solid than in a mobile liquid. For Cyclohexane, the phase transition used, at $6.55^{\circ} \mathrm{C}$ in the bulk, is between a plastic crystal phase and the liquid. Near the phase transition the plastic phase has a transverse nuclear magnetisation decay time $\mathrm{T}_{2}$ of about $2 \mathrm{~ms}$, while that of the bulk liquid is of the order of a few seconds.

If $T_{2}$ is longer than the coherence decay time $T_{2}{ }^{*}$ of the magnet, this measurement of 
the amount of liquid is usually most conveniently made using a $90^{\circ}{ }_{x}-\tau$ - $180^{\circ}{ }^{-}-\tau$-echo sequence $^{[3]}$, where the time interval $2 \tau$ is set to be longer than the decay time in the solid but less than the decay time in the liquid, and is typically around $20 \mathrm{~ms}$. Thus the amplitude of the echo records the amount of solid that has melted to a liquid at a particular temperature.

The echo amplitude is then differentiated as a function of temperature, and re-mapped according to the Gibbs-Thomson equation, to obtain a pore size distribution. i.e. porosity measured as a function of pore diameter.

We have been developing this technique in our laboratories ${ }^{[2,4]}$, and have recently combined it with magnetic resonance imaging techniques to obtain spatially resolved pore size distributions. Thus we demonstrate here the use of nuclear magnetic resonance both as a convenient technique for measuring pore size by determining the fraction that is liquid, inside the porous material, and as a way of spatially encoding this pore size information, to produce a pore size distribution map.

\section{Experimental arrangement}

\section{$2.1 \quad 1 \mathrm{D}$ Measurements}

For the 1D measurements, a spectrometer developed for bulk Cryoporometric measurements was used. This was driven from a personal computer (PC) running a Labview $^{T M}$ for Windows ${ }^{T M}$ program written by us, and was interfaced by IEEE488 to a Gould ${ }^{T M} 1604$ oscilloscope and a Hewlett Packard ${ }^{T M} 34401 \mathrm{~A}$ multimeter, and by serial interface to a Control Techniques ${ }^{T M} 452+$ proportional-integral-derivative temperature controller. The solid state NMR relaxation spectrometer used was designed and constructed in our laboratory.

The magnet was a $21.5 \mathrm{MHz}$ (protons) Mullard ${ }^{T M}$ permanent magnet, with a $35 \mathrm{~mm}$ gap, into which was inserted a Dewar containing the NMR probe, with cooling by direct injection of liquid nitrogen ${ }^{[5]}$. The sample size was a standard $5 \mathrm{~mm}$ high resolution NMR sample tube.

\section{$2.2 \quad 2 \mathrm{D}$ and $3 \mathrm{D}$ Measurements}

For the two-dimensional and three-dimensional measurements, the spectrometer used was a modified Bruker ${ }^{T M}$ CXP200 console with a Doty Scientific $^{T M}$ DSI1000A 1kW Class AB R.F. power amplifier and three Techron ${ }^{T M} 7700$ series gradient amplifiers.

Rather than employing the usual Bruker Aspect ${ }^{T M}$ computer, the Bruker was interfaced by us to an industrial grade 486 DX33 personal computer running SMIS $^{\text {} M}$ software and with SMIS plug in modules including an AT\& TM $^{\text {тM }}$ DP32C digital signal processor. This interface was designed using an Altera ${ }^{T M}$ EPM5128 programable gate array, programmed to mimic the action of the Aspect device bus interface (20 bit data $+8+3+2$ controls), and driven by 11 bits of a parallel port from the PC ${ }^{[6]}$. It provided control of the Bruker frequency synthesizer, frequency sweep unit, modulator 
control, filter control and output interface.

Gating and phase selection of the Bruker R.F. was from the software controlled pulse sequencer in the SMIS system, using the remaining 5 bits of the parallel port. A four quadrant R.F. multiplier was introduced before the $20 \mathrm{~W}$ broadband amplifier, and controlled from an analogue channel of the SMIS sequencer, programmed to generate a sinc $\left(t / T_{0}\right)$ function of width $T_{0}$, to provide soft pulses for slice selection.

Sixth order Butterworth equal capacitor Salen and Key filters ${ }^{[7]}$ with cut-off frequencies of $100 \mathrm{kHz}, 300 \mathrm{kHz}$ and $1 \mathrm{MHz}$ (plus unfiltered) were constructed and introduced in both receiver channels, for use at higher bandwidths than the Bruker filters.

The R.F. and gradient coils were designed and constructed for imaging broadline materials $^{[6,8,9,10]}$. The R.F. coil was of birdcage construction; the $\mathrm{z}$ gradient along the main magnetic field $\mathrm{B}_{0}$ was generated by a solenoidal Maxwell pair, and the $\mathrm{x}$ and $\mathrm{y}$ gradients were generated by Golay saddle coils.

The magnet used was a $200 \mathrm{~mm}$ horizontal bore Magnex ${ }^{T M}$ superconducting magnet, operating at $105 \mathrm{MHz}$ for protons. The sample access diameter (room temperature, inside the gradient and R.F. coils) was $25 \mathrm{~mm}$. Into this was inserted the narrow neck of a dewar, about $100 \mathrm{~mm}$ long, with $12.5 \mathrm{~mm}$ internal diameter clear bore, that then swelled out (allowing hand access) and extended to the edge of the magnet.

\section{1D NMR Cryoporometry}

Test samples were prepared (Fig. 1a), in $5 \mathrm{~mm}$ diameter tubes, each consisting of three layers of porous silica of $60 \AA, 140 \AA$ and $500 \AA$ nominal pore diameter, separated by PTFE spacers (giving no proton signal). Water or cyclohexane was added to the silica, to give approximately $110 \%$ filling of the pores.

To obtain spatial resolution of the pore size distribution phase and/or frequency encoding of the NMR signal ${ }^{[11]}$ may used. In the case of $1 \mathrm{D}$ Cryoporometry ${ }^{[4]}$, the main $\mathrm{B}_{0}$ field was applied along the $\mathrm{z}$ axis, and a constant magnetic field gradient $\mathrm{G}_{y}$ $=\delta \mathrm{B}_{z} / \delta \mathrm{y}$ was applied in the perpendicular $\mathrm{y}$ direction along the axis of the NMR sample tube, to obtain frequency encoding of the axial position. The gradient was generated by four current carrying wires plus return wires, suitably spaced ${ }^{[12,13]}$ to minimise all but the linear gradient term $\mathrm{G}_{y}$.

Since the NMR precession frequency is proportional to the magnetic field $\mathrm{B}_{0}$, the frequency of the NMR signal varied linearly with axial position. The space between the magnet pole faces (34mm pole gap) and the existing dewar was limited, thus the gradient set was constructed from four one-turn rectangular coils cut from copper sheet, and mounted on drafting film. A DC current of $7 \mathrm{~A}$ produced a suitable gradient of $0.05 \mathrm{~T} \cdot \mathrm{m}^{-1}$.

Rather than recording just the peak amplitude of the echo at each temperature as in normal NMR cryoporometry, the complete echo envelope from a $90^{\circ}{ }_{x}-\tau$ - $180^{\circ}{ }_{y}$ - $\tau$-echo sequence was captured. Fig. 2 shows the particularly simple sequence, with the static gradient $\mathrm{G}_{y}=\delta \mathrm{B}_{z} / \delta \mathrm{y}$. Since the linear field-gradient allows a linear mapping from 
frequency space to y-dimension, the Fourier transform of the echo in the gradient then gives a 1D profile of the liquid component at this temperature.

The sample temperature was measured with a copper/constantan thermocouple soldered to a grounded copper foil wrapped round the sample, providing temperature uniformity, inside the R.F. coil. (Provided the foil does not form a closed turn it also aids R.F. homogeneity, and hence the ability to obtain precise $90^{\circ}$ and $180^{\circ}$ precession throughout the sample volume).

To make a measurement the test sample was cooled until all the liquid was frozen, and there was no signal. It was then slowly allowed to warm naturally in the gradient. A succession of 1D profiles was captured, as the sample warmed. Initially all the sample was frozen, and there was no signal. The liquid in the $60 \AA$ silica melted first, then that in the $140 \AA$ silica, followed by that in the $500 \AA$ silica, and finally any bulk liquid outside the pores.

When the temperature run was complete, the data was analysed as follows. First the oscilloscope traces were Fourier transformed, and then the standard cryoporometric differentiation and re-mapping according to the Gibbs-Thomson equation was performed along the temperature axis, for each array point in the sample.

This re-mapped data is presented as a surface map in Fig. 1b, giving the porosity within the sample as a function of pore diameter and axial position, and as a colour map (porosity and pore diameter map to luminance and hue) in Fig. 1c.

These show that the porosity is successfully resolved with good spatial resolution and with pore size resolution comparable with that from a standard cryoporometry run. The median pore size was also calculated, for each axial point in the 1D sample (Fig. $1 \mathrm{~d})$, showing good agreement with the nominal pore sizes.

\section{2D Resolved Pore Size Distributions}

A test phantom (Fig. 3) was constructed, consisting of four $5 \mathrm{~mm}$ high-resolution thin walled glass tubes, containing $40 \AA, 60 \AA, 140 \AA$, and $200 \AA$ pore diameter porous silica, arranged in a square array.

Anhydrous cyclohexane was added to the dried silica in a glove box under a nitrogen atmosphere, and the tubes evacuated and flame sealed.

The samples were inserted into the neck of the dewar. Silicon grease was used to keep them in thermal contact with a cylinder of pyrophyllite, used as an insulating thermal mass. A copper/constantan thermocouple was embedded in a hole in the pyrophyllite cylinder, to allow the temperature to be monitored. The neck of the dewar was filled with foam, to provide additional thermal insulation.

The sample was cooled with liquid nitrogen, and 32 images were recorded as a function of temperature, as the phantom warmed up. At each temperature, a 2-dimensional cross-sectional image of the liquid was obtained using Fourier reconstruction. A single slice of $20 \mathrm{~mm}$ was selected along the $\mathrm{Z}$ direction of the magnetic field using soft pulses. This slice was positioned so as to exclude the silicon grease from the image.

The imaging sequence used was modified from being a gradient echo sequence (Fig. 
4a), to one using a $90^{\circ}{ }^{-}-\tau$ - $180^{\circ}{ }^{-}-\tau$-echo sequence (Fig. $4 \mathrm{~b}$ ), with a $2 \tau$ of $14 \mathrm{~ms}$, as this time was greater than the $\mathrm{T}_{2}{ }^{*}$ of the wide-bore magnet used. The $\mathrm{X} Y$ phasing ensures that the echo maintains the same relative phase as the FID. Here X and Y are understood to cycle round through all combinations of $\pm \mathrm{X}, \pm \mathrm{Y}$ as required by the CYCLOPS sequence, preserving their relative orientation.

CYCLOPS phase cycling ${ }^{[14]}$ was used to eliminate D.C. offsets and ghosting due to receiver channel gain differences. The CYCLOPS sequence rotates the basic sequence used through $0^{\circ}, 90^{\circ}, 180^{\circ}, 270^{\circ}$ phase offsets: a change of $180^{\circ}$ inverts the phase of the signal, thus by subtracting the $\pm \mathrm{X}$ and $\pm Y$ signals $\mathrm{I}_{X}-\mathrm{I}_{-X}, \mathrm{I}_{Y}-\mathrm{I}_{-Y}$, one removes any D.C. offset (and hence zero frequency spikes in the image); similarly a rotation of $90^{\circ}$ swaps the real and imaginary signals $\Re(\mathrm{I}), \Im(\mathrm{I})$ between the receiver $\mathrm{U}$ and $\mathrm{V}$ channels, thus by adding the resultant $\mathrm{X}, \mathrm{Y}$ signals one eliminates ghosting due to receiver $\mathrm{U}$ and $\mathrm{V}$ channel gain (A) differences; finally one has $\Re(\mathrm{I}) \cdot 2 \cdot\left(\mathrm{A}_{U}+\mathrm{A}_{V}\right)$, $\Im(\mathrm{I}) \cdot 2 \cdot\left(\mathrm{A}_{U}+\mathrm{A}_{V}\right)$.

Averaging was kept to the minimum of 4 required by the CYCLOPS sequence, giving a slice imaging time of 4.3 minutes, with a repeat time of 1 second limited by $T_{1}$.

Data was captured as one read frequency encode (4 times oversampled), and one phase encode, for a resolution of $128 \times 32$, but reconstructed into 32 maps of $64 \times 64$ images. Fourier reconstruction of the $2 \mathrm{D}$ data sets for each temperature was performed on the Digital Signal Processor of the SMIS console PC.

32 images were recorded as a function of temperature, as the phantom warmed up. The resulting $\mathrm{N}_{X} \times \mathrm{N}_{Y} \times \mathrm{N}_{T}$ data set was stored as 32 maps of $64 \times 64$ integer images, which occupies 0.5 MByte for the temperature run. This data set was then transferred to an 4 MByte Atari ${ }^{T M}$ running Mint multitasking and Apl68000 ${ }^{T M}$, but no floating point accelerator.

Selected images are shown in Fig. 5, where the sequential melting of the liquid in the $40 \AA, 60 \AA, 140 \AA$ and $200 \AA$ pore diameter silicas can be seen as the image signal of the liquid.

Applying the constraint along the temperature axis that a noiseless signal should only increase in amplitude as the temperature increases and the sample melts (monotonic), gave a marked improvement in signal to noise (Figs. 5, 6). This is particularly important as the next step in the analysis is differentiation along the temperature axis.

The 32 images (averaged in pairs for display purposes) are shown in Fig. 7. The cryoporometric differentiation with respect to temperature and re-map according to the Gibbs-Thomson equation was then applied to each xy pixel of the data set in Fig. 7. The resulting porosity maps as a function of pore diameter and xy position are shown in Fig. 8.

Routines were written to plot the poresize distribution as a set of angled planes, both to paint on the screen and to generate postscript. A naive implementation of the above 18 slice picture would have generated a postscript file 4.4MByte in size; the final one produced is $420 \mathrm{kByte}$, saving creation and printing time in proportion.

It is possible to pick individual pixels from the data structure of Fig. 8, and plot the 
full pore size distributions for these pixels. This has been done for the four pixels $(22,22)(22,42)(42,22)(42,42)$ (Fig. 9). Pore size resolution is comparable with that obtained with standard cryoporometry runs, except at large pore sizes where it can be improved by warming more slowly.

A plot of median pore size for each pixel in Fig. 8 has also been generated (Fig. 10). This provides a concise summary of the average pore sizes present, though containing less information. A colour map of pore size is also shown (Fig. 11).

\section{3D Resolved Pore Size Structure}

A test phantom was prepared in a $10 \mathrm{~mm}$ diameter tube, that visually appears to be just $15 \mathrm{~mm}$ of silica powder in the bottom of the tube.

The sample was actually prepared, using dry $60 \AA$ and $500 \AA$ nominal pore diameter silica as follows : about $5 \mathrm{~mm}$ of $60 \AA$ silica was placed in the bottom of the $10 \mathrm{~mm}$ tube, then $20 \mathrm{~mm}$ of $60 \AA$ silica was placed in a $5 \mathrm{~mm}$ tube, which was then up-ended and pushed to the base of the $10 \mathrm{~mm}$ tube. $500 \AA$ silica was added as a collar, to a further depth of about $5 \mathrm{~mm}$.

The $5 \mathrm{~mm}$ tube was then carefully withdrawn, while tapping, hopefully leaving a central column of $60 \AA$ silica, the excess then spilling over the $500 \AA$ silica, to a further depth of about $5 \mathrm{~mm}$. The ideal shape of the $500 \AA$ and $60 \AA$ silica, ignoring mixing of the dry powders, hand shake, etc. is given in Fig. 13a. The phantom was then fully dried, and anhydrous cyclohexane added to the silica under dry nitrogen in a glovebox.

The phantom was cooled, and 3D image sets captured as a function of temperature. The imaging sequence used (Fig. 12) was a 3D Fourier transform sequence (one read frequency encode, and two phase encodes), for a resolution of $128 \times 32 \times 32$, but reconstructed into $(64 \times 64) \times 32$ slices at each temperature. Again a spin echo sequence was used, with a $2 \tau$ of $14 \mathrm{~ms}$, with CYCLOPS phase cycling.

Each 3D image data set took about 60 minutes to acquire, with a repeat time of 1 second. As with the $2 \mathrm{D}$ case, improvements are required in the control of the sample temperature. Another problem is the quantity of data required if one wishes for full poresize distributions throughout a $3 \mathrm{D}$ volume, as one has to capture a data set $\mathrm{N}_{X}$ $\times \mathrm{N}_{Y} \times \mathrm{N}_{Z} \times \mathrm{N}_{T}$ in size.

For the purposes of this study $\mathrm{N}_{T}$ was restricted to 2, one measurement in a temperature range where the cyclohexane in the $60 \AA$ silica was liquid, but that in the $200 \AA$ silica was frozen $\left(250^{\circ} \mathrm{K}\right.$ to $\left.265^{\circ} \mathrm{K}\right)$, the other at room temperature. Strictly the latter measurement should have been made just below the bulk melting point of the cyclohexane, to exclude bulk liquid, but the temperature control was not sufficiently precise.

A thermal time-constant of some hours, when liquid $\mathrm{N}_{2}$ was injected into the dewar, facilitated the lower temperature measurement.

The frequency axis was oriented longitudinally, to aid axial positioning of the sample in the magnet bore. 
Fourier reconstruction of the 3D data sets for each temperature was performed on the digital signal processor of the SMIS console. The resulting $\mathrm{N}_{X} \times \mathrm{N}_{Y} \times \mathrm{N}_{Z}$ data set at each temperature was set at $64 \times 32 \times 64$, stored as 32 slices of $64 \times 64$ integer images, which occupies 0.5 MByte for each temperature.

A 3-dimensional data set was then obtained for the $500 \AA$ silica on its own, using Apl. The measured data sets were first brought into register (there was noticeable displacement along the frequency axis). The data set intensities were compared with suitable amplitudes, and a 3D bit array generated for each temperature, and then the following tri-state logical operation performed :

$$
\text { Silica500 } \leftarrow 1=(\text { Warm }- \text { Cold })
$$

This bit data set, when constructed as a shaded 3D image, gives a well separated image, but shows the abrupt steps in the logical bits very clearly. The bit data set can be used in a multiplicative operation to select the relevant intensities from the original Warm integer data set. Finally a 3D three point smoothing was applied, that performs a Gaussian-like three point smoothing along each of the principle axies, and all of the 3D diagonals. This then generated a 3D data set that the surface rendering could operate on with a minimum of artifacts.

$3 \mathrm{D}$ rendering was performed using routines written at our laboratory ${ }^{[15]}$. These routines can be compiled for any $\mathrm{Unix}^{T M}$ host, and were run on a PC running Linux, with XV as a viewing window. The images were generated into a $512 \times 512$ window, and cropped. Three point 2D smoothing was applied, with a 24 bit colour table.

Colour postscript images were generated (Fig. 13b). The left hand image is of all the silica powder, with no discrimination. The middle image shows just the $500 \AA$ silica, the right hand image is of just the $60 \AA$ silica.

It is possible to view the $3 \mathrm{D}$ data sets from any direction; a view from above the sample shows the collar of $500 \AA$ silica more clearly (Fig. 13c).

This ability to take a porous solid and to generate images of just those regions whose pore sizes fall within a particular range is a novel capability that must have wide applications in physical, chemical and biological studies.

\section{Conclusion}

A method for determining the full pore size distribution and median pore size for any point in a 1 or 2-dimensional sample has been presented. Measurement and shaded surface representation of 3-dimensionally resolved pore size structures has also been demonstrated.

This method offers a uniquely non-destructive method of obtaining the full poresize distribution or median pore size at any point inside a bulk sample. 


\section{$7 \quad$ References}

[1] Jackson, C.L. and McKenna, G.B., J. Chem Phys. 93, 9002-9011 (1990).

[2] Strange, J.H., Rahman, M. and Smith, E.G., Phys. Rev. Let. 71, 21, 3589-3591, (1993).

[3] Harris, R.K., Nuclear Magnetic Resonance Spectroscopy, A Physicochemical View. Longman Scientific \& Technical. (1987).

[4] Strange, J.H. and Webber, J.B., Characterisation of Porous Solids by NMR, 12th Specialised Colloque Ampere, Corfu 1995.

[5] Norris, M.O. and Strange, J.H., A Nuclear Magnetic Resonance sample temperature controller using liquid nitrogen injection. J.Phys. E, 2, 2, 1106-1108, (1969).

[6] Macnair, A., A Targetted System for High Resolution in Vivo Broad-Line MRI, Ph.D. Thesis, University of Kent, (1995).

[7] Aikens, R.S. and Kerwin, W.J., Single Amplifier, Minimal RC, Butterworth, Thomson, and Chebyshev filters to sixth order. Active RC filters: Theory and Application, Ed. Huelsman, L.P. Dowden, Hutchinson \& Ross, Inc., John Wiley \& Sons, Inc., (1976)

[8] Hayes, C.E., Scheneck, J.F., Mueller, O.M., and Eash, M., An efficient, highly homogeneous radiofrequency coil for whole-body NMR imaging at 1.5T. J.Mag. Reson., 63, 622-628, (1985).

[9] Strange, J.H. and Halse, M.R., Imaging Techniques for Solids and Quasi-solids. Encyclopaedia of NMR, 2472-2481, Wiley, ISBN 0-471 958 395, (1996).

[10] Codd, S.L., 3DFT NMR Imaging of Solid-Like Materials, Ph.D. Thesis, University of Kent, (1996).

[11] Callaghan, P.T., Principles of Nuclear Magnetic Resonance Microscopy. Oxford Science Publications, Clarendon Press, Oxford, (1993).

[12] Kroon, D.J., Laboratory Magnets. Philips Technical Library, Eindhoven, 186-189, (1968).

[13] Rahman. H.J., M.R.I. Studies of Polymeric Systems, Ph.D. Thesis, University of Kent, (1991).

[14] Hoult, D.I., Richards, R.E., Proc. Roy. Soc. (London) A344, 311, (1975).

[15] Green, T.A.P., Three Dimensional Visualization in NMR Imaging, M.Sc. Thesis, University of Kent, (1994).

\section{Acknowledgements}

The authors thank EPSRC, Unilever Research, Oxford Instruments AIG and SMIS Ltd. for research support. 


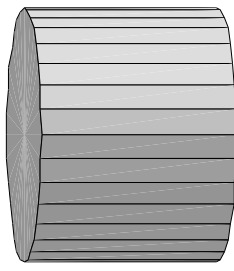

$500 \AA$ Silica

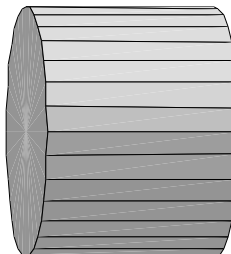

$140 \AA$ Silica

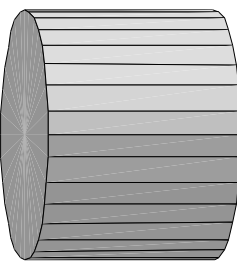

$60 \AA$ Silica

(a:) $1 D$ porous silica phantom with axial structure in the $y$ direction.

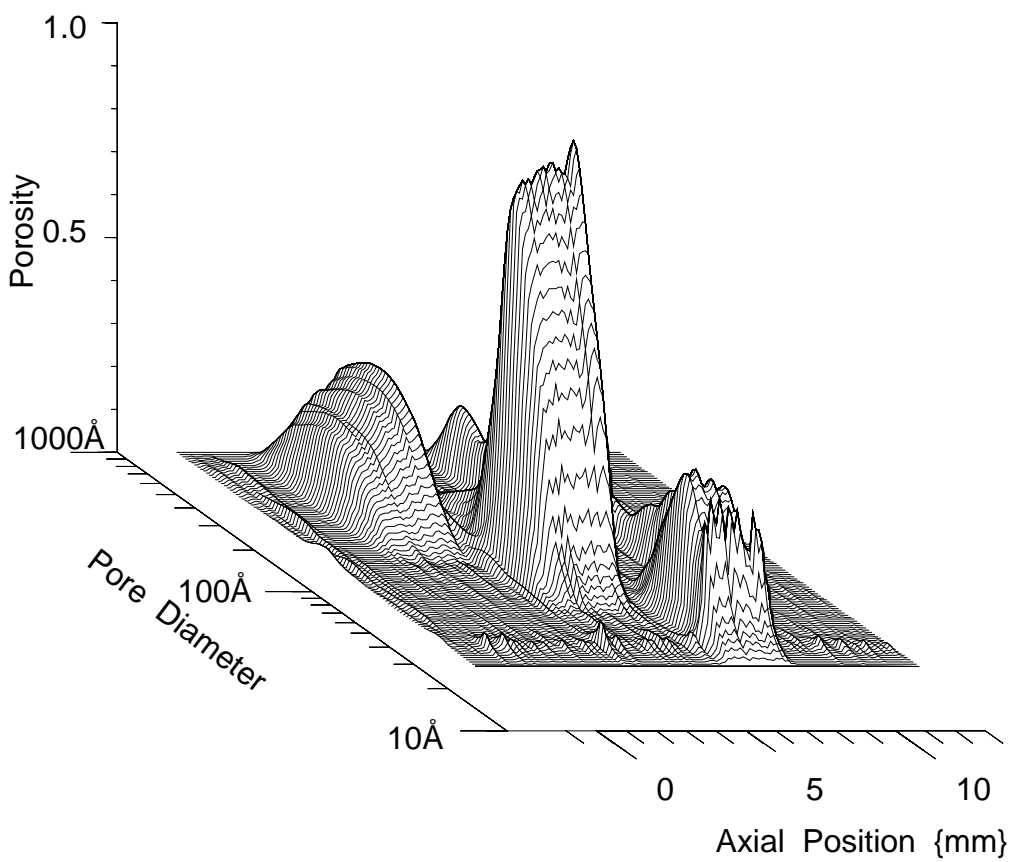

(b:) $1 D$ resolved porosity for 3 part phantom.
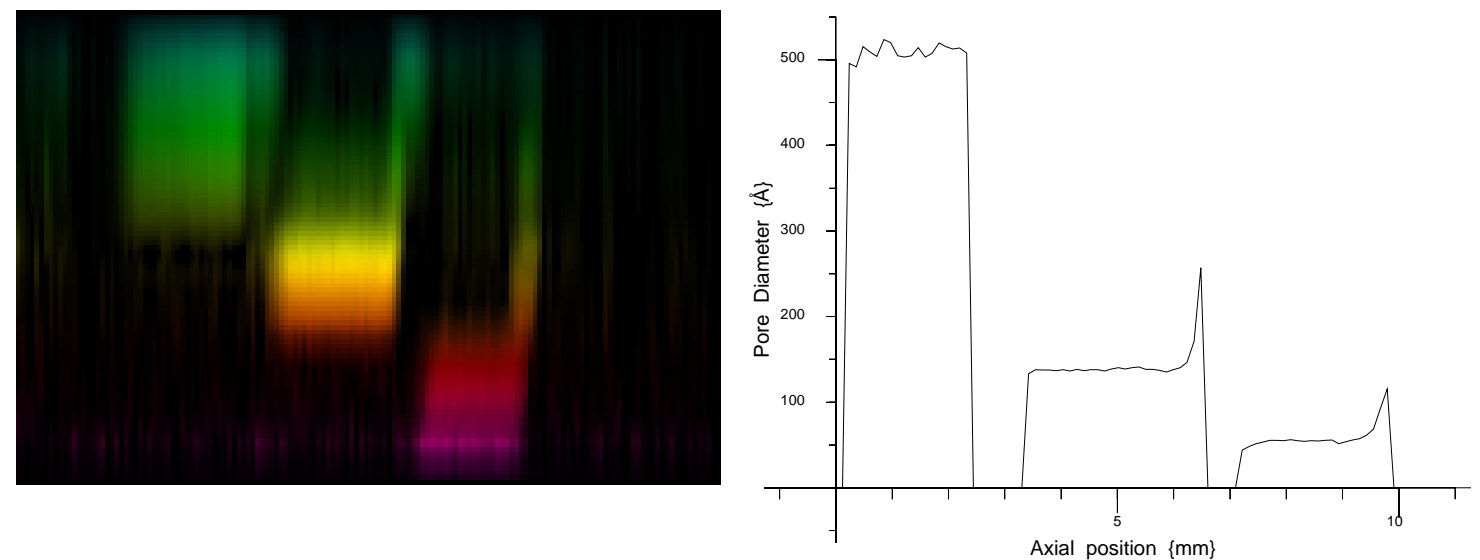

(c:) Colour map of Pore Size.

(d:) Median pore size as a function of axial position, for the phantom constructed from nominal 500A, $140 \AA$ and $60 \AA$ silicas.

Figure 1: 1D Cryoporometry. 


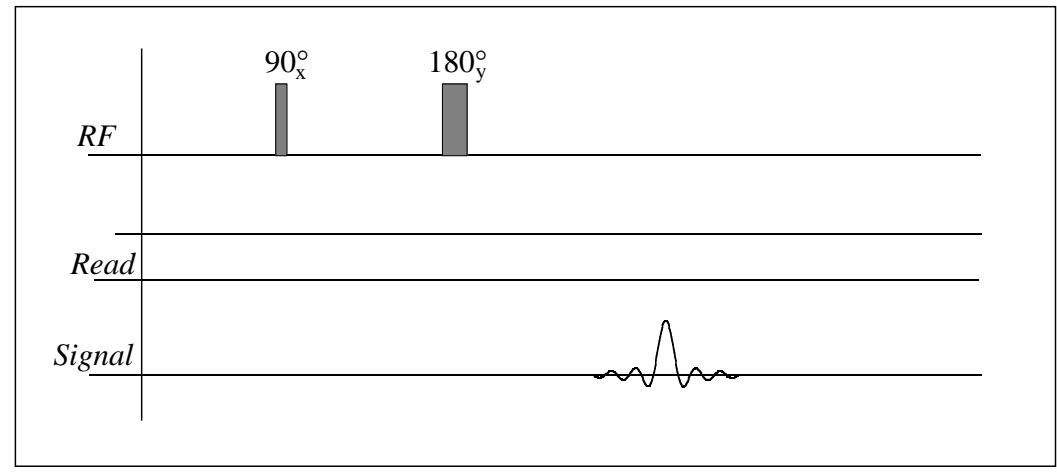

$1 D$ spin echo sequence with static read gradient $G_{y}=\delta B_{z} / \delta_{y}$.

Figure 2: 1D imaging sequence.

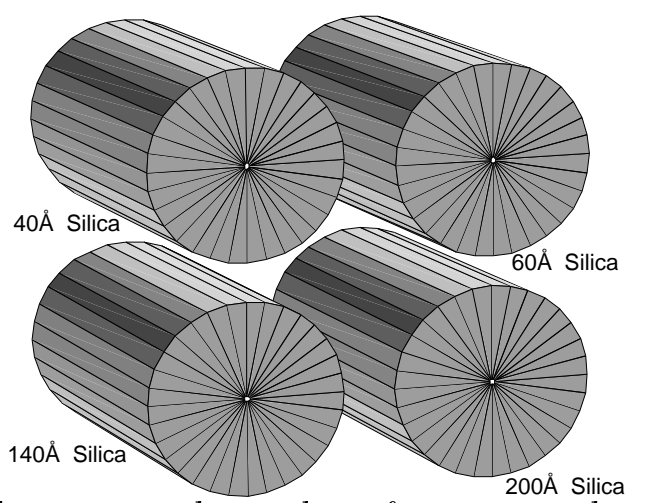

$2 D$ silica phantom with axial uniformity in the $z$ direction.

Figure 3: 2D Cryoporometry sample with xy porous structure 


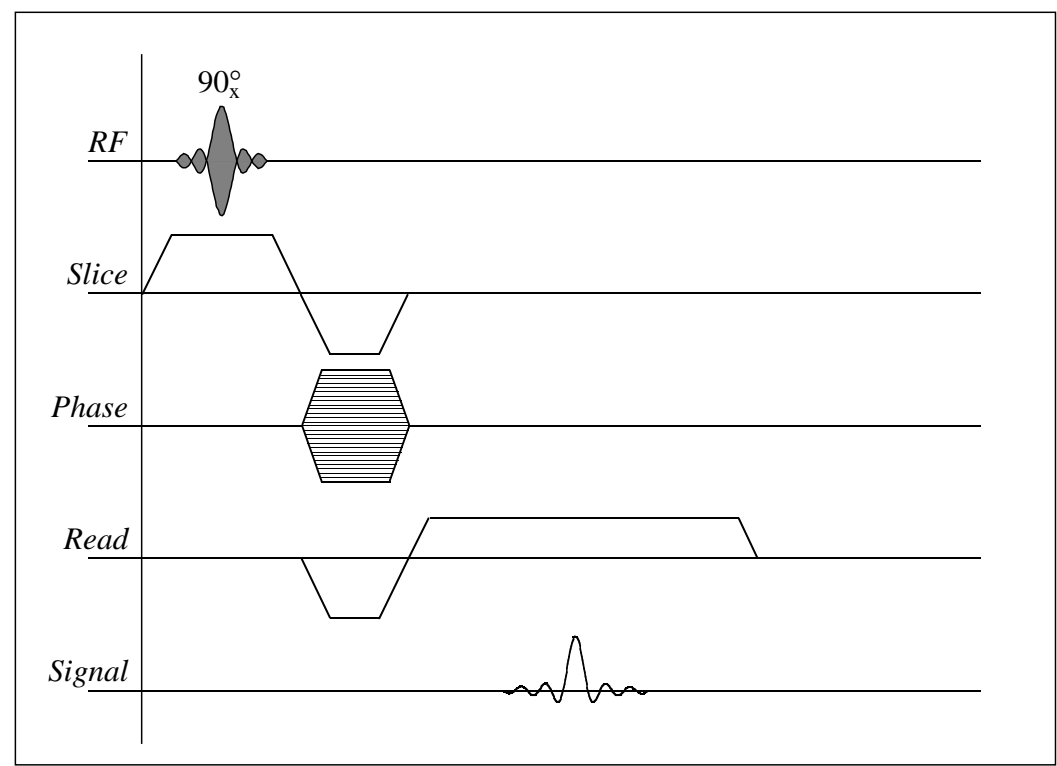

a) $2 D$ gradient echo sequence.

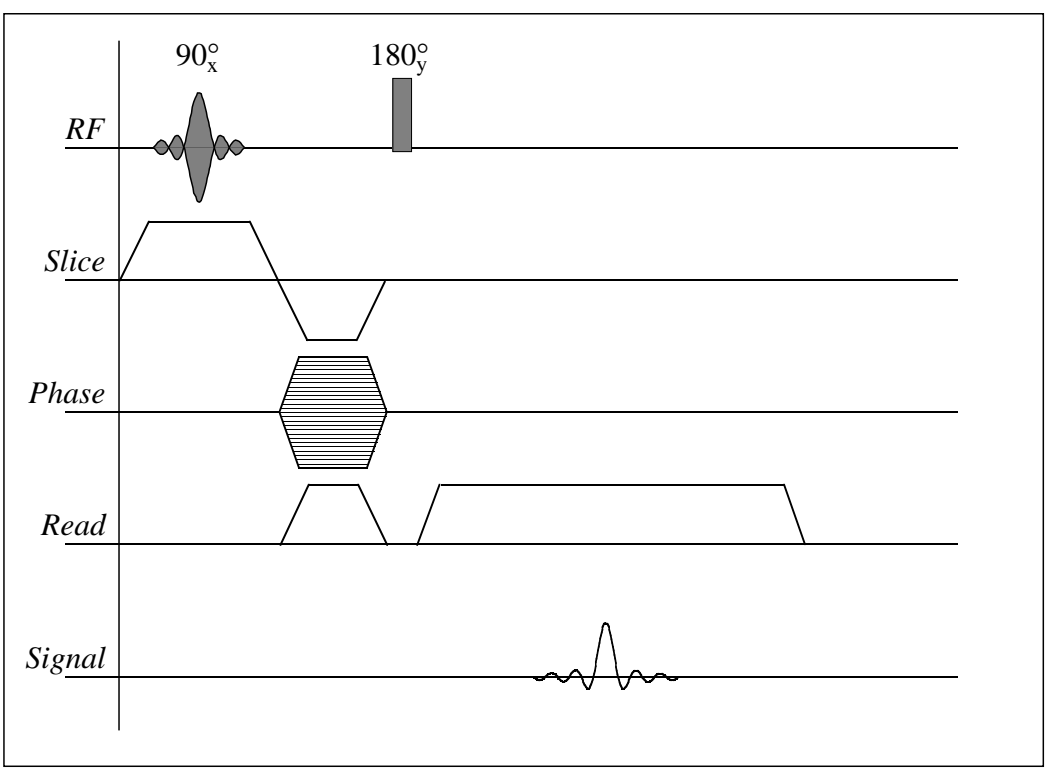

b) $2 D$ spin echo sequence with slice select gradient $G_{z}=\delta B_{z} / \delta_{z}$, phase encode gradient $G_{y}=\delta B_{z} / \delta_{y}$, and read gradient $G_{x}=\delta B_{z} / \delta_{x}$.

Figure 4: 2D imaging sequences. 

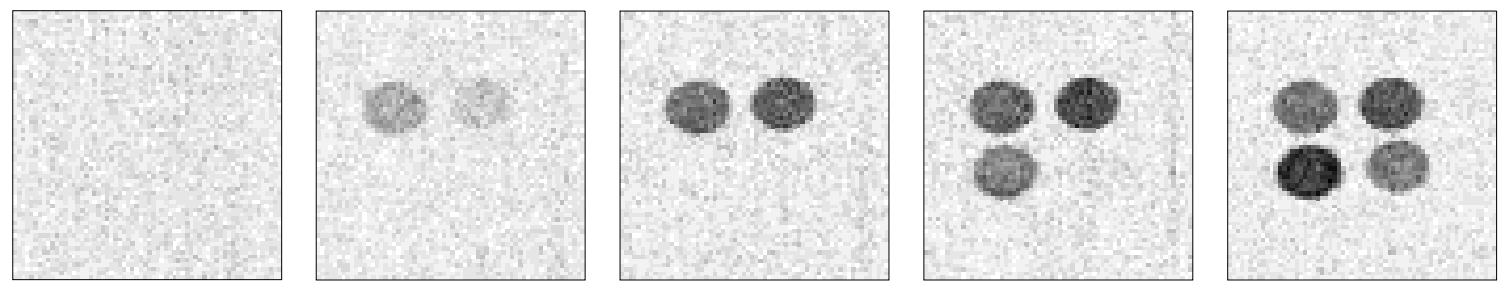

Map 2: $-135^{\circ} \mathrm{C} \quad$ Map 9: $-38^{\circ} \mathrm{C}$ Map 12: -220 $\mathrm{C}$ Map 16: $-6^{\circ} \mathrm{C}$ Map 20: $5^{\circ} \mathrm{C}$ Raw intensity maps showing the sequential melting of the liquid in the 40A, $60 \AA$, $140 \AA$ and $200 \AA$ pore diameter silicas.

Figure 5: Selected intensity maps from the temperature run.
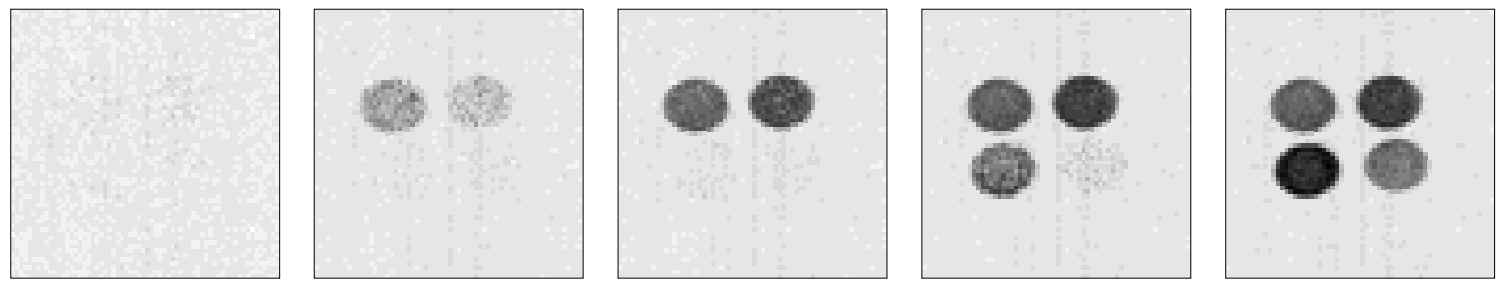

Map 2: $-135^{\circ} \mathrm{C}$ Map 9: $-38^{\circ} \mathrm{C}$ Map 12: $-22^{\circ} \mathrm{C}$ Map 16: $-6^{\circ} \mathrm{C}$ Map 20: $5^{\circ} \mathrm{C}$ Improved signal to noise after application of the constraint of monotonicity in melting.

Figure 6: Monotonic intensity maps from the temperature run. 


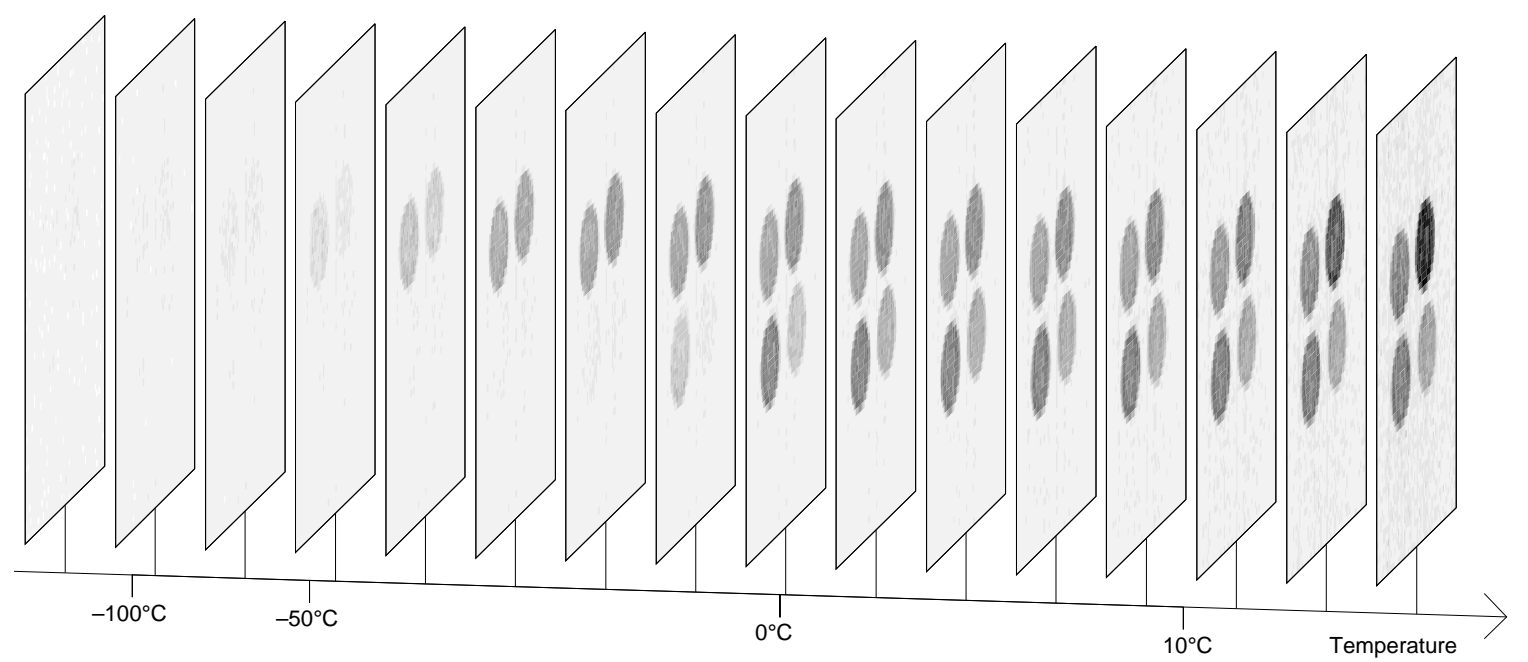

Liquid proton density images showing the melting of the liquid in larger pore sizes at higher temperatures.

Figure 7: Liquid Proton Density images vs. Temperature.

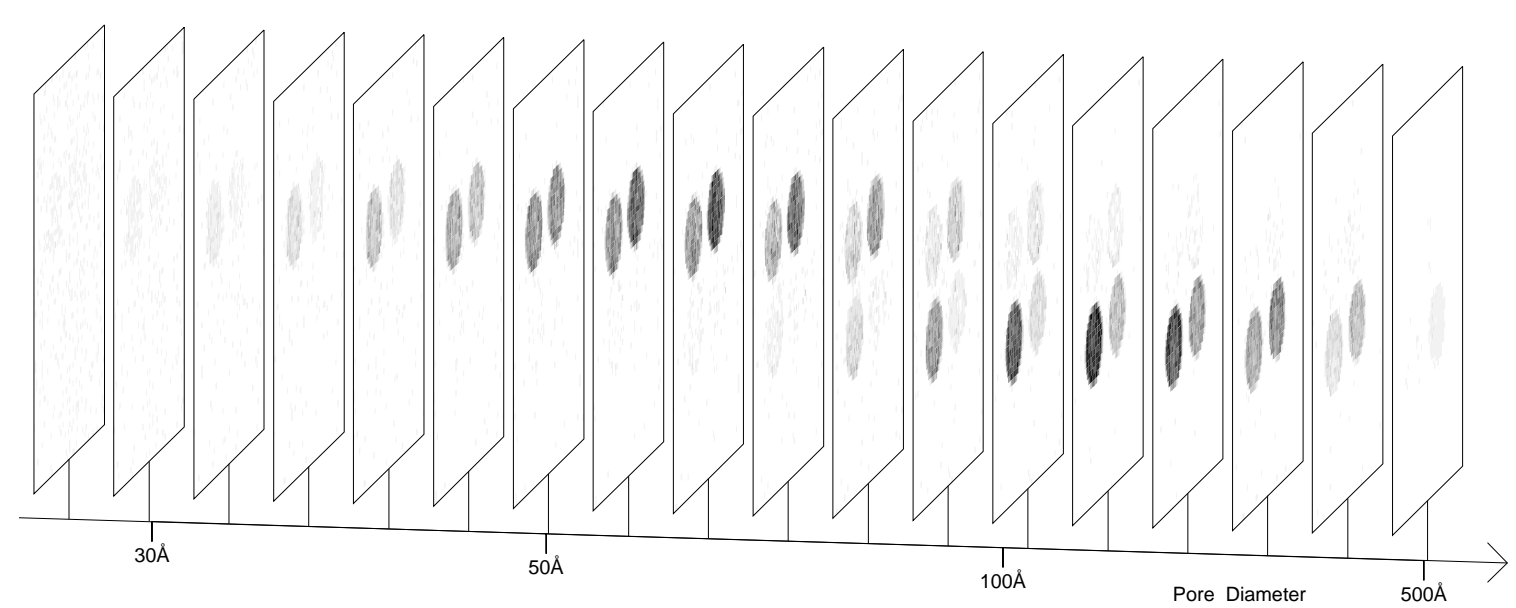

Porosity resolved as a function of pore diameter and xy location, obtained from Fig. 7 by cryoporometric differentiation with respect to temperature and re-mapping.

Figure 8: Porosity maps vs. Pore Diameter. 

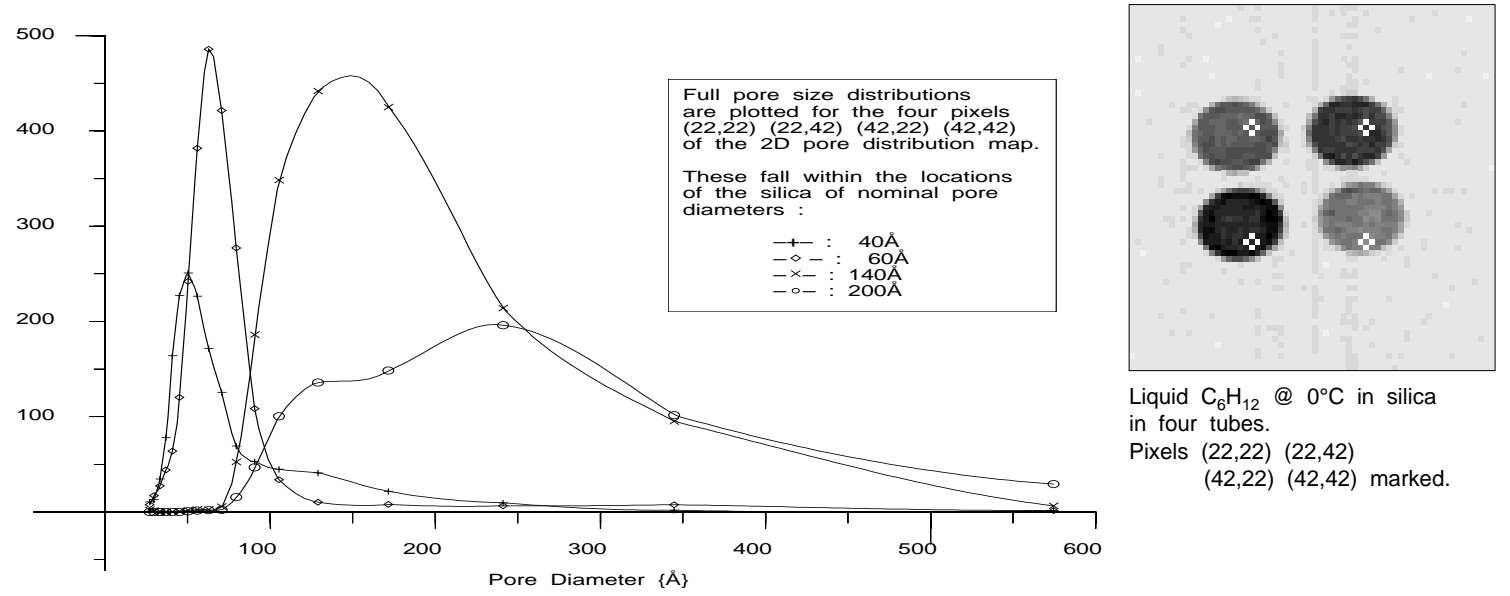

Liquid $\mathrm{C}_{6} \mathrm{H}_{12} @ 0^{\circ} \mathrm{C}$ in silica in four tubes.

Pixels $(22,22)(22,42)$

$(42,22)(42,42)$ marked

4 Pore Size Distributions from 2D Map

Localised pore size distributions are extracted from the data in Fig. 8, for four pixels. The peaks of the distributions agree well with the nominal pore sizes.

Figure 9: Localised Pore Size Distributions.

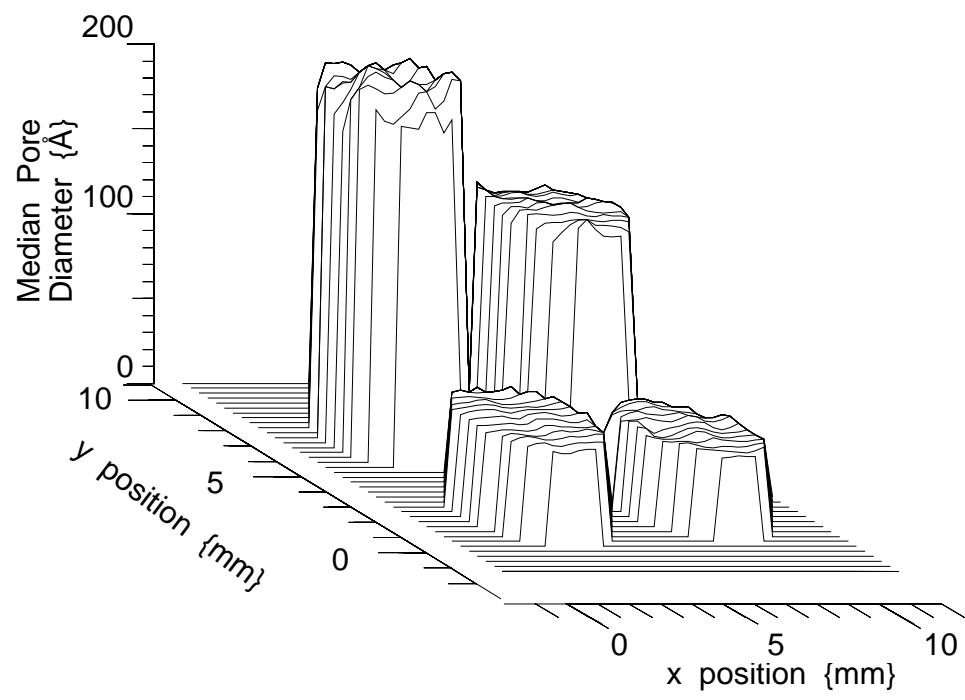

A map of the Median Pore Size for the four tube phantom, rotated for ease of viewing).

Figure 10: 2D map of Median Pore Size. 


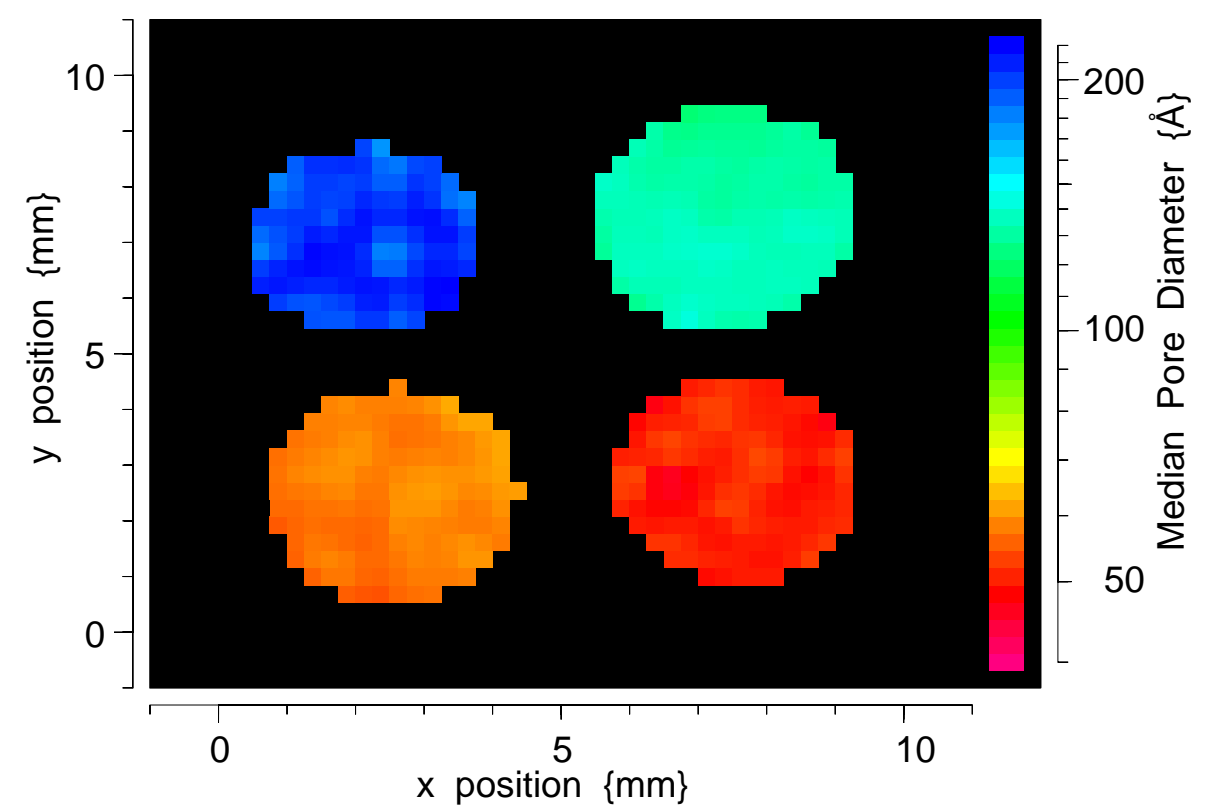

$\log _{10}$ Median Pore Diameter mapped to Hue, for the four tube phantom. Blue : 200A, Cyan : 140A, Orange : $60 \AA$, Red : $40 \AA$.

Figure 11: Colour map of Median Pore Size.

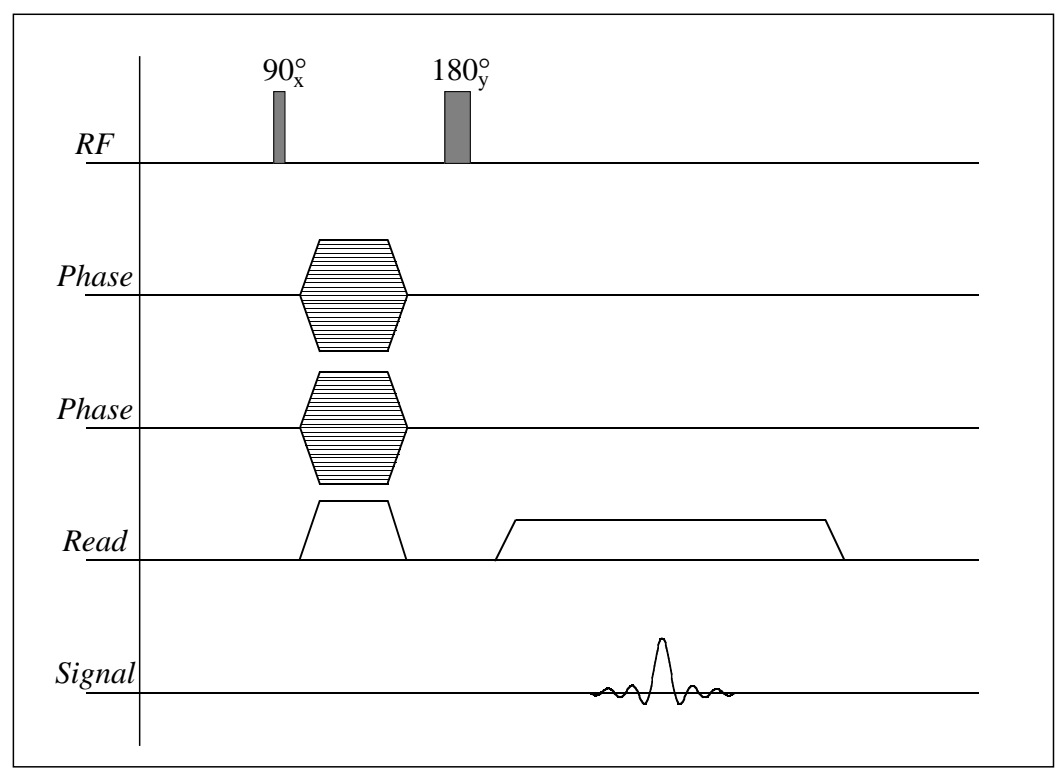

$3 D$ spin echo sequence with phase encode gradients $G_{x}=\delta B_{z} / \delta_{x}$ and $G_{y}=\delta B_{z} / \delta_{y}$, and read gradient $G_{z}=\delta B_{z} / \delta_{z}$.

Figure 12: 3D imaging sequence. 


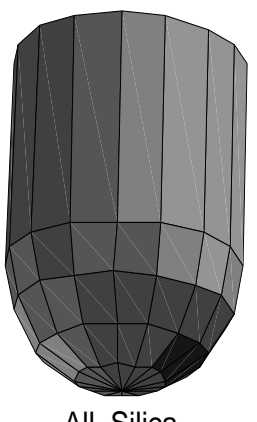

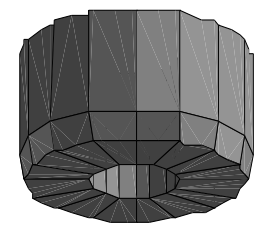

$500 \AA$ Silica

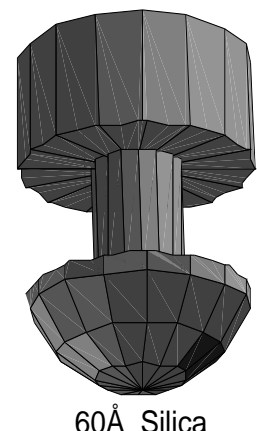

$60 \AA$ Silica

(a:) Idealised $3 D$ Structure of $500 \AA$ plus $60 \AA$ silica phantom.

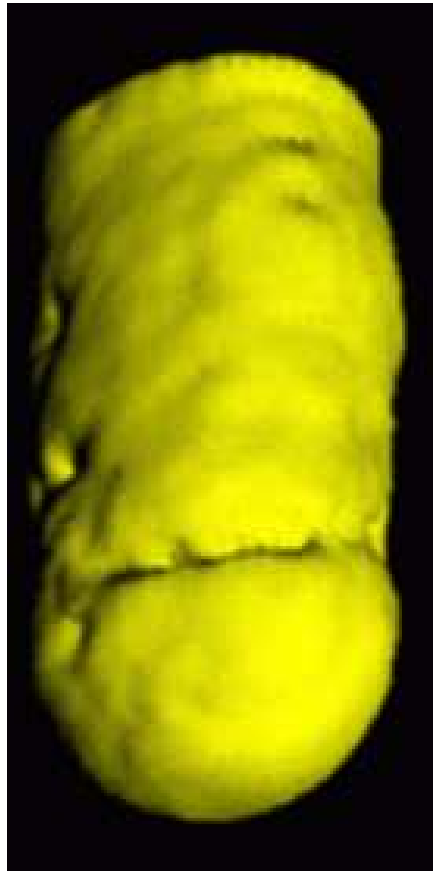

Undifferentiated Silica

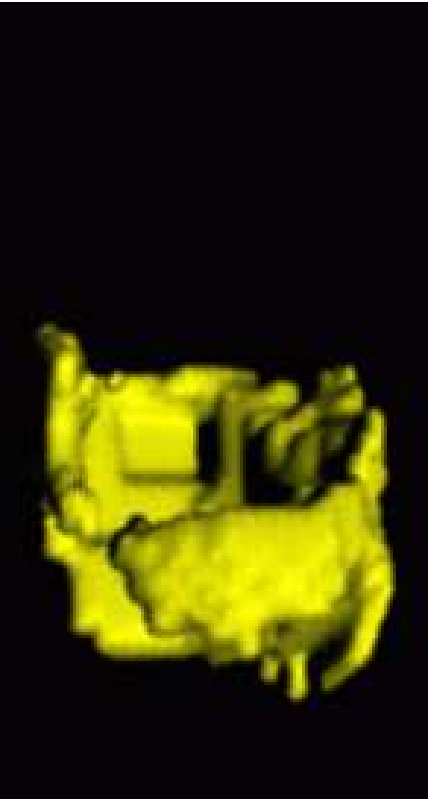

$500 \AA$ Silica

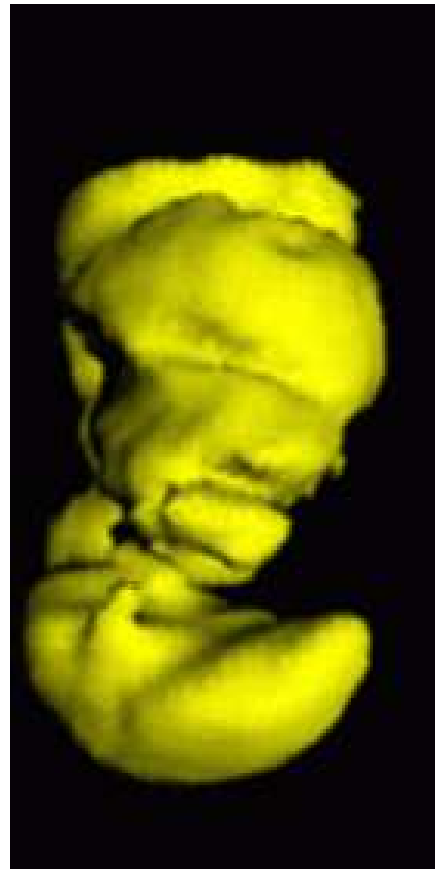

$60 \AA$ Silica

(b:) $3 D$ resolved Pore Size Structure.

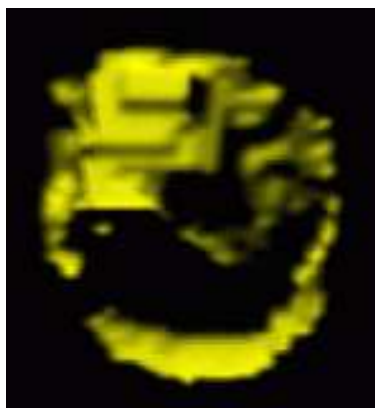

(c:) Top view of $500 \AA$ Silica.

Figure 13: 3D porous sample resolved into $500 \AA$ and $60 \AA$ components 